\title{
Especies de Thysanoptera asociadasa viñedos en la zona centro norte de la provincia de Córdoba (Argentina)
}

Viglianco, A. I., Cragnolini, C. I., Salvo, A. y Avalos, D. S.

DOI 10.31047/1668.298x.v38.n2.32486

\begin{abstract}
RESUMEN
Los tisanópteros constituyen uno de los grupos de insectos que limitan la producción de los viñedos al dañar bayas y hojas por acción de su aparato bucal. El objetivo de este trabajo fue determinar las especies encontradas en viñedos comerciales y su abundancia relativa en las diferentes etapas fenológicas del cultivo en Colonia Caroya, provincia de Córdoba, Argentina. Para la identificación de las especies de trips se recolectaron ejemplares en cinco viñedos comerciales y de cinco variedades de vid entre 2004 y 2007. Para analizar la abundancia poblacional de adultos y larvas en los estados fenológicos de la vid, en 2009 y 2010 se realizaron muestreos en la variedad Malbec de un viñedo comercial. Se identificaron cuatro especies de tisanópteros presentes en flores, con predominio de Frankliniella gemina. El pico de abundancia de adultos ocurrió en plena floración mientras que el de larvas se observó entre los estados de fin de floración y cuajado de frutos.
\end{abstract}

Palabras clave: Trips, Frankliniella gemina, variedades de vid

Viglianco, A. I., Cragnolini, C. I., Salvo, A. and Avalos, D. S. 2021. Thysanoptera species associated with vineyards in the north-central area of the province of Córdoba (Argentina). Agriscientia 38 (2): 135-141

\section{SUMMARY}

Thrips are one of the insect groups that limit the production of grapevines by damaging berries and leaves through their mouthparts. The aim of this study was to determine the species found on commercial vineyards and their relative abundance in the different phenological stages of the grapevine in Colonia Caroya, Córdoba province, Argentina. For the identification of thrips species, specimens were collected from five commercial vineyards and five grapevine varieties between 2004 and 2007. To analyze the abundance of adults and larvae in relation to the phenological stages of grapevine, sampling was carried out in 2009 and 2010 in a commercial vineyard of Malbec. Four 
species of thysanopterans were identified on flowers, with predominance of Frankliniella gemina. The peak of adult abundance occurred in full bloom while that of larvae was observed between the end of flowering and the fruit set stages.

Key words: Thrips, Frankliniella gemina, grapevine varieties

Viglianco, A. I. (ORCID: 0000-0002-2310-078X), Cragnolini, C. I. (ORCID: 0000-0003-0221-9754) y Avalos, D. S. (ORCID: 0000-0002-8898-0810): Universidad Nacional de Córdoba, Facultad de Ciencias Agropecuarias. Terapéutica Vegetal, Córdoba, Argentina. Salvo, A. (ORCID: 0000-00025487-7794): Universidad Nacional de Córdoba, Facultad de Ciencias Exactas, Físicas y Naturales, Centro de Investigaciones Entomológicas de Córdoba del Instituto Multidisciplinario de Biología Vegetal (IMBIV). Correspondencia a: aviglian@agro.unc.edu.ar

\section{INTRODUCCIÓN}

La vid (Vitis vinifera L., Vitaceae) con 7,4 millones de hectáreas cultivadas (Organización Internacional de la Viña y el Vino, 2018) se puede considerar el frutal (Agusti, 2004; Pearson y Goheen, 2007) de mayor distribución mundial, ya que se cultiva en todos los continentes excepto en la Antártida (Hidalgo Fernández-Cano y Hidalgo Togores, 2011).

En Argentina este cultivo abarca una superficie de 223944 ha, de las cuales el 92,2\% corresponden a variedades de uvas tintas. Entre las provincias argentinas, Córdoba ocupa el octavo lugar en cuanto a superficie cultivada (309 ha), y de ésta, el 59 \% se encuentra en Colonia Caroya y alrededores (Departamento Colón), destacándose la producción de diversas variedades de $V$. vinifera (como Malbec, Merlot, Pinot Noir, Cabernet Sauvignon, Ancelota, Chardonnay, Sauvignon Blanc) y una de V. labrusca (Isabella). El 95\% del total de la superficie implantada con viñedos de esta provincia registra variedades aptas para elaboración de vinos y/o mostos, predominando las tintas (86\%), seguidas de las blancas (12\%) (Instituto Nacional de Viticultura, 2020).

Entre los insectos perjudiciales que limitan la producción de la vid se mencionan diferentes especies de los órdenes Hemiptera, Lepidoptera y Thysanoptera (Pearson y Goheen, 2007; Pavan et al., 2014). Los representantes de este último orden, conocidos comúnmente como "trips", ocasionan diferentes perjuicios debido a su modo de alimentación, provocando daños directos o indirectos. Los daños indirectos ocasionados por trips en plantas se asocian a la transmisión de virosis (Kirk, 1997), aunque no existen reportes de transmisiones virósicas por trips en vid. Numerosas citas señalan a los tisanópteros como perjudiciales en viñedos; sin embargo, en la región sur del continente americano son poco estudiados (Mound, 2014). Los registros de las especies de trips presentes en el género Vitis son escasos en Argentina, al igual que en Córdoba.

Se señala a Drepanothrips reuteri Uzel ("trips europeo de la vid") como el trips más frecuente en hojas, mientras que sobre inflorescencias y bayas se ha citado a Frankliniella occidentalis (Pergande) o "trips oriental de las flores" (Yokohama, 1977a; 1977b; Özsemerci et al., 2011) y a Thrips tabaci Lindeman (Özsemerci et al., 2011).

En Brasil, Chile y Uruguay se han registrado las siguientes especies de trips en las flores de cultivos de uvas de mesa: F. occidentalis (Mujica et al., 2007; Araya et al., 2014; Moreira et al., 2014); Heliothrips haemorrhoidalis (Bouché) (Pinent et al., 2005); varias especies de Frankliniella como F. gemina Bagnall, F. gardeniae Moulton, F. schultzei (Trybom), F. brevicaulis Hood (Moreira et al., 2012; Moreira et al., 2014); F. serrata Moulton (Formolo et al., 2011); además de F. cestrum Moulton y T. tabaci (Ripa et al., 1992). Por otro lado, Moreira et al. (2012) identifican cuatro especies en hojas de vid: H. haemorrhoidalis, Retithrips syriacus Mayet, Selenothrips rubrocinctus (Giard) y F. schultzei.

Los escasos estudios realizados en Argentina hasta el momento han permitido identificar seis 
especies asociadas a la vid: T. tabaci, F. australis Morgan, F. occidentalis, D. reuteri (de Borbón y Herrera, 2012), F. juancarlosi (de Borbón y Zamar, 2018) y F. gemina (Viglianco et al., 2005; de Borbón, 2013). Considerando los antecedentes expuestos, el objetivo del trabajo fue conocer las especies presentes en viñedos, en la zona centro norte de la provincia de Córdoba y su abundancia relativa en los diferentes estados fenológicos del cultivo.

\section{MATERIALES Y MÉTODOS}

El estudio se llevó a cabo en dos etapas: la primera desde 2004 a 2007 para la identificación de las especies y la segunda durante 2009-2010, para determinar la fluctuación en la abundancia poblacional de larvas y adultos en relación al estado fenológico (EF) de la vid, de acuerdo a Eichhorn y Lorenz (1977).

En la primera etapa se trabajó en cinco viñedos comerciales ubicados en Colonia Caroya $\left(31^{\circ} 02^{\prime}\right.$ 00" S, 64 05' 00" O, a 509 m s.n.m., Departamento Colón) y con cinco variedades de vid, cuatro de la especie V. vinifera (Malbec, Merlot, Pinot Noir, Chardonnay) y una de V. labrusca (Isabella). En cada uno de los viñedos y para cada variedad, semanalmente y al azar se tomaron dos tipos de muestras: muestras de diez hojas (una por planta, todas con igual probabilidad de ser seleccionadas independientemente de su tamaño o ubicación), desde que cada planta tenía cinco o seis hojas extendidas (EF12) hasta el estado de uvas maduras para cosecha (EF 38); y muestras de diez fragmentos de inflorescencias $(3$ a $4 \mathrm{~cm}$ ) desde el inicio de floración (EF 19) hasta el estado de bayas pequeñas (4 mm de diámetro) (EF 29). El material recolectado se colocó en bolsas plásticas y se mantuvo en heladera hasta el momento de ser analizado.

En la segunda etapa, para determinar la fluctuación de la abundancia de larvas y adultos de trips se seleccionó la variedad Malbec en uno de los cinco viñedos comerciales antes citados. Se tomaron semanalmente y al azar, cuatro muestras de diez fragmentos de inflorescencias ( 3 a $4 \mathrm{~cm}$, una por planta, todas con igual probabilidad de ser seleccionadas), desde el estado fenológico de floración temprana (EF 21) hasta el de bayas pequeñas (EF 29).

Los insectos extraídos del material vegetal se colocaron en AGA (alcohol etílico 60\% + glicerina + ácido acético en proporciones de 10:1:1). Posteriormente se realizó el montaje de adultos y larvas según metodología propuesta por Mound y Kibby (1998). Para la identificación específica de los trips se utilizaron claves taxonómicas (Mound y Kibby, 1998; Monteiro, 1999; de Borbón, 2007; Cavalleri y Mound, 2012; de Borbón, 2013).

La abundancia relativa de cada especie de trips presente en el género Vitis y en cada variedad de vid se estimó como el número de trips/flor, incluyendo en el conteo de insectos tanto adultos como larvas, mediante la siguiente fórmula:

$$
\operatorname{Ar}=\mathrm{n} / \mathrm{N} \times 100
$$

Donde: $\operatorname{Ar}(\%)$ : Abundancia relativa

n: número de individuos de cada especie

$\mathrm{N}$ : Número total de individuos de la muestra

Dado que el patrón de la fluctuación de abundancia de adultos y larvas en ambos años fue similar, la variación temporal en los diferentes estados fenológicos de Malbec se graficó considerando el número promedio de adultos y larvas (trips/flor) colectados en 2009 y 2010.

\section{RESULTADOS Y DISCUSIÓN}

Se colectaron e identificaron un total de 6759 ejemplares de trips provenientes de fragmentos de inflorescencias ( $n$ total $=414$ inflorescencias) de las cinco variedades de vid evaluadas (Merlot $=104$; Malbec $=80$ Chardonnay $=54$; Pinot Noir $=78$ e Isabella =98). Según se observa en la Tabla 1 se registraron cuatro especies de Thysanoptera. De las cuatro especies encontradas en este estudio, tres de ellas, T. tabaci, F. occidentalis, F. gemina, ya han sido registradas en el país (Viglianco et al., 2005; de Borbón y Herrera, 2012; de Borbón, 2013); en tanto que la observación de F. schultzei en flores de vid se realiza por primera vez en Argentina. Hasta el momento sólo fue citada en Brasil, sobre flores y hojas de vid (Moreira et al., 2012; Moreira et al., 2014). La riqueza específica de trips observada en los viñedos estudiados fue similar a otros registros realizados en el continente americano, donde se mencionan entre dos y nueve especies, dependiendo del país (Ripa et al., 1993; Moreira et al., 2012; Moreira et al., 2014). Sin embargo, son notablemente menores a lo observado en diferentes países de otros continentes, por ejemplo, en viñedos de Turquía

Tabla 1. Especies de Thysanoptera registradas en flores de vid en Colonia Caroya, durante el período 2004 a 2007

Familia Sub-familia Género Especie

Thripidae Thripinae Frankliniella gemina Bagnall, 1919

schultzei(Trybom, 1910)

occidentalis (Pergande)

Thrips tabaci Lindeman1889 
donde se han reportado entre 19 (Kaplan et al., 2016) y 35 especies (Özsemerci et al., 2011).

El $99,08 \%$ de los individuos recolectados correspondió a F. gemina y menos del 1\% del total, a las restantes especies (Tabla 2). El notable predominio de F. gemina fue similar en todas las variedades de vid. F. schultzei y T. tabaci, se encontraron en todas las variedades analizadas, mientras que $F$. occidentalis estuvo ausente en una de ellas (Tabla 2). El total de larvas $(n=150)$ provenientes de flores de las diferentes variedades fueron identificadas como F. gemina y no se encontraron adultos ni larvas de trips en hojas $(n=1026)$. Nuestros resultados concuerdan con lo hallado en vides de Brasil donde F. gemina (considerada sinónimo de Frankliniella rodeos Moulton por Cavalleri y Mound, 2012), se ha observado con un predominio del $98 \%$, en período de floración (Formolo et al., 2011; Moreira et al., 2014). Esta especie, de origen y distribución sudamericana (De Santis et al., 1980), ha sido registrada en Argentina (Viglianco et al., 2005; de Borbón, 2013), Brasil (Cavalleri y Mound, 2012) y Perú (Ortiz, 1977). Es antófila y ampliamente polífaga, mayormente asociada a dicotiledóneas (Monteiro, 2002), tanto a frutales (de Borbón, 2013; Soares Matos et al., 2019) como a cultivos hortícolas (de Borbón, 2005).

En concordancia con lo aquí registrado, el fuerte predominio de una única especie de trips en flores de vid fue observado en viñedos de Uruguay (Mujica et al., 2007), Nueva Zelanda (Marroni et al., 2012) y Sudáfrica (Allsopp, 2010). La dominancia de F. gemina en flores de vid en la zona de estudio puede deberse a diferentes razones. En primer lugar, F. gemina es la única especie de origen y distribución sudamericana (De Santis et al., 1980; Mound y Marullo, 1996), por lo que resulta esperable que las características ambientales de la región de estudio favorezcan su potencial reproductivo y le confieran mayor aptitud competitiva que a las demás especies aquí registradas (Kirk, 2002). Por otra parte, al haberse detectado la presencia tanto de adultos como de larvas de F. gemina en los viñedos de Colonia Caroya podría asumirse que las flores y frutos recién cuajados constituyen una dieta muy nutritiva para esta especie, favoreciendo el desarrollo de su ciclo. Se ha demostrado que la cantidad de insectos inmaduros en un hospedador proporciona una medida de la aceptabilidad de la planta como huésped reproductor (Terry, 1997). Teniendo esto en cuenta, es posible que las vides analizadas no constituyan hospederos de calidad adecuada para F. occidentalis, T. tabaci y F. schultzei en la región de estudio, y que su presencia haya resultado transitoria o accidental.

Las restantes tres especies registradas se caracterizan por ser antófilas, folívoras y polífagas (Hoddle et al., 2012; de Borbón, 2013). F. occidentalis es nativa de Norteamérica, con fuerte impacto en la agricultura a nivel mundial (Reitz, 2009). En tanto que F. schultzeies de origen incierto, probablemente originaria de América del Sur o de África (Hoddle et al., 2012). Contrariamente a lo observado en Córdoba, en viñedos de Uruguay la abundancia de F. occidentalis fue superior al $99 \%$ y coincidió con el escaso número de ejemplares de F. schultzei (Mujica et al., 2007). Thrips tabaci, posiblemente originaria del este mediterráneo, se asocia usualmente a los géneros de Liliaceae (Hoddle et al., 2012), Fabaceae, Asteraceae y Brassicaceae (De Santis et al., 1980; Monteiro, 2002). Esta especie de aparición esporádica en los viñedos de la zona de estudio, ha sido señalada como dominante en otros países (Ripa et al., 1992; Merket al., 2006). Su escasa presencia en flores de vid de Colonia Caroya podría deberse a su preferencia por otras familias botánicas cultivadas o espontáneas, presentes en la zona.

Para la determinación de la abundancia de trips se recolectaron 3855 adultos y 288 larvas en 2009, y 1873 adultos y 1008 larvas en 2010. Al considerar el número promedio de adultos de ambos años se observó un pico de abundancia en el estado de plena floración (EF 23) mientras que el pico de abundancia de larvas ocurrió entre los estados de

Tabla 2. Abundancia relativa y total de cada especie de trips por variedad de vid y en el total de inflorescencias evaluadas en Colonia Caroya (Córdoba) en el período 2004-2007

\begin{tabular}{|c|c|c|c|c|c|c|}
\hline \multirow{2}{*}{ Especie de trips } & \multicolumn{5}{|c|}{ Variedades de vid } & \multirow{2}{*}{$\begin{array}{c}\% \text { Total } \\
(\mathrm{N})\end{array}$} \\
\hline & Chardonnay & Merlot & Malbec & Pinot Noir & Isabella & \\
\hline F. gemina & $\begin{array}{l}98,78 \\
(838)\end{array}$ & $\begin{array}{c}99,47 \\
(2265)\end{array}$ & $\begin{array}{c}99,24 \\
(1946)\end{array}$ & $\begin{array}{l}99,05 \\
(938)\end{array}$ & $\begin{array}{l}97,93 \\
(710)\end{array}$ & $\begin{array}{c}99 \% \\
(6697)\end{array}$ \\
\hline F. schultzei & $\begin{array}{c}1,02 \\
(9)\end{array}$ & $\begin{array}{c}0,21 \\
(5)\end{array}$ & $\begin{array}{l}0,15 \\
(3)\end{array}$ & $\begin{array}{l}0,21 \\
(2)\end{array}$ & $\begin{array}{c}0,83 \\
(6)\end{array}$ & $\begin{array}{c}0,37 \% \\
(25)\end{array}$ \\
\hline F. occidentalis & $\begin{array}{c}0,00 \\
(0)\end{array}$ & $\begin{array}{l}0,11 \\
(3)\end{array}$ & $\begin{array}{l}0,25 \\
(5)\end{array}$ & $\begin{array}{c}0,42 \\
(4)\end{array}$ & $\begin{array}{c}0,69 \\
(5)\end{array}$ & $\begin{array}{c}0,25 \% \\
(17)\end{array}$ \\
\hline T. tabaci & $\begin{array}{c}0,20 \\
(2)\end{array}$ & $\begin{array}{c}0,21 \\
(4)\end{array}$ & $\begin{array}{c}0,36 \\
(7)\end{array}$ & $\begin{array}{c}0,32 \\
(3)\end{array}$ & $\begin{array}{c}0,55 \\
(4)\end{array}$ & $\begin{array}{c}0,30 \% \\
(20)\end{array}$ \\
\hline
\end{tabular}




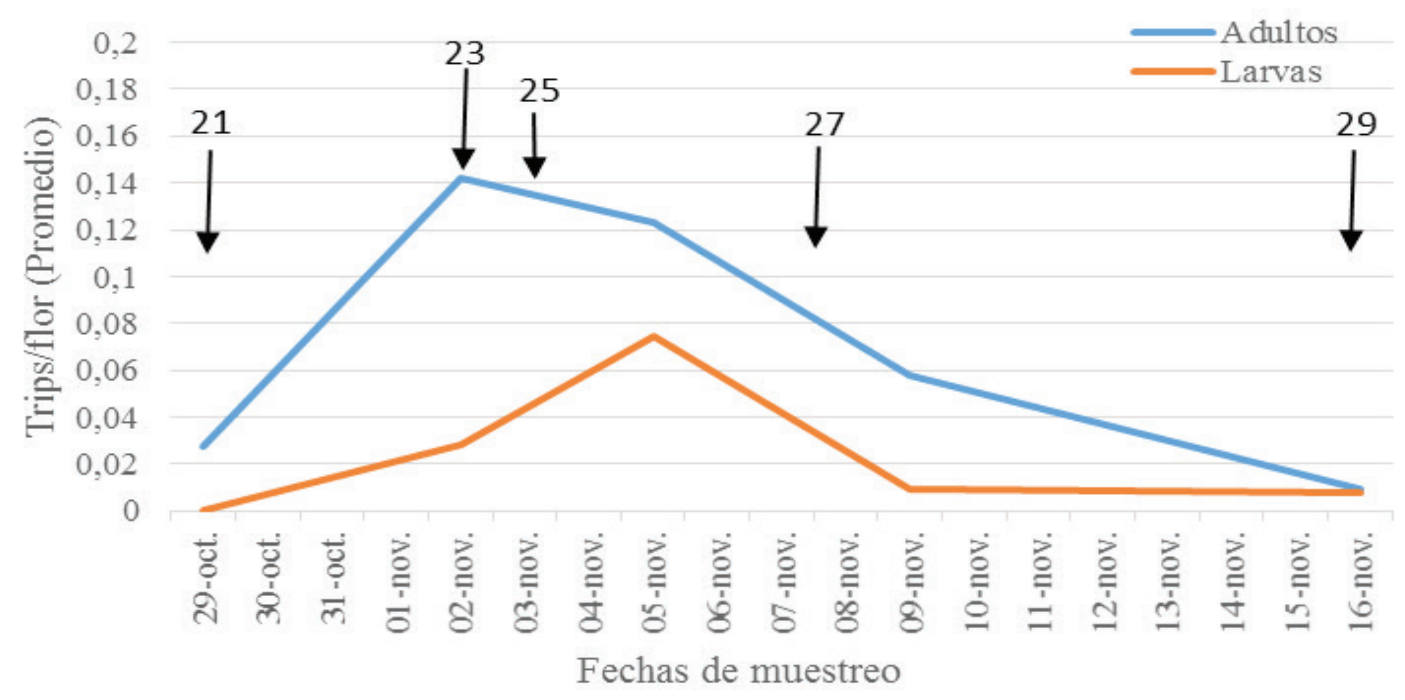

Figura 1. Variación temporal de la abundancia de trips/flor en los diferentes estados fenológicos (21, 23, 25, 27 y 29 siguiendo a Eichhorn y Lorenz [1977], indicados con flechas) de la variedad Malbec en Colonia Caroya en 2009 y 2010

fin de floración (EF 25) y cuajado de frutos (EF 27) (Figura 1).

A diferencia de otros estudios que señalan la presencia de diferentes especies de trips durante todo el ciclo del cultivo (Moleas y Addante, 1993; Özsemerci et al., 2011), nuestros resultados indican que la presencia de adultos y larvas se restringe al período comprendido entre la floración y el inicio de fructificación. Por otra parte, en casos en que el cultivo fue atacado en todas las fases fenológicas, el período de floración se ha señalado como el de mayor infestación (Moreira et al., 2012).

El período de mayor captura de adultos en flores de Malbec coincidió con los estados de plena floración y fin de floración (caída de caliptra) y luego disminuyó, probablemente relacionado con la disminución de la calidad del alimento en el cultivo. Esta observación coincide con los registros de diferentes especies de trips en viñedos de Uruguay (Mujica et al., 2007), Chile (Ripa et al., 1993), Brasil (Moreira et al., 2012), Nueva Zelanda (Marroni et al., 2012) y Sudáfrica (Allsopp, 2010). Por otra parte, a partir de fin de la floración (EF 25) se observó un incremento de la población de larvas, alcanzando su máxima densidad entre este estado fenológico y cuajado de frutos (EF 27). Similar comportamiento fue observado para $F$. occidentalis (Ripa et al., 1993) y T. tabaci en flores de vid (Jensen y Luvisi, 1973).
En Colonia Caroya (provincia de Córdoba, Argentina), F. geminaes la especie predominante de Thysanoptera. La presencia de adultos y larvas en las etapas reproductivas del cultivo (abundancias máximas de adultos en plena floración y de larvas al momento de cuajado de frutos), la posiciona como una especie potencialmente perjudicial para los viñedos de la región. A los fines de corroborar su importancia económica, futuras investigaciones deberían abordar el impacto que ocasionan sus daños.

\section{AGRADECIMIENTOS}

Los autores agradecen al Ing. Agr. Carlos de Borbón (Inta EEA Mendoza) por la identificación de las especies de trips. Este estudio ha sido parcialmente financiado por la Secretaría de Ciencia y Técnica de la Universidad Nacional de Córdoba (SECyT-UNC), Córdoba.

\section{BIBLIOGRAFÍA}

Agustí, M. (2004). Fruticultura. Ediciones Mundi-Prensa.

Allsopp, E. (2010). Seasonal occurrence of Western flower thrips, Frankliniella occidentalis (Pergande), on Table Grapes in the Hex River Valley, South Africa. South African Journal for Enology and Viticulture, 31(1), 49-57. https://doi.org/10.21548/31-1-1400

Araya, J. E., Merino, C., Santibáñez, F. y Sazo, L. (2014). Manchas por alimentación de Frankliniella occidentalis 
(Thysanoptera: Thripidae) en uva de mesa blanca. Revista Colombiana de Entomología, 40(1), 1-6.

Cavalleri, A. y Mound, L. A. (2012). Toward the identification of Frankliniella species in Brasil (Thysanoptera, Thripidae). Zootaxa, 3270(1), 1-30. https://doi.org/10.11646/zootaxa.3270.1.1

de Borbón, C. M. (2005). Los trips del suborden Terebrantia de la provincia de Mendoza. Instituto Nacional de Tecnología Agropecuaria.

de Borbón, C. M. (2007). Clave para la identificación del segundo estadío larval de algunos trips comunes (Thysanoptera: Thripidae). Mendoza, Argentina. Revista de la Facultad de Ciencias Agrarias UNCuyo, 39(1), 69-81.

de Borbón, C. M. (2013). Especies del género Frankliniella (Thysanoptera: Thripidae) registradas en Argentina, una actualización. Revista de la Facultad de Ciencias Agrarias UNCuyo, 45(1), 259-284.

de Borbón, C. M. y Herrera, M. E. (2012). El trips cazador negro, Leptothrips mali (Fitch) en viñedos mendocinos. Trece grados, 25, 20-23.

de Borbón, C. M. y Zamar, M. I. (2018). Two new species of Frankliniella (Thysanoptera: Thripidae) from Argentina with a key to species from Argentina and Chile, Zootaxa, 4369(3), 419-431. https://doi.org/10.11646/ zootaxa.4369.3.7

De Santis, L., Gallego de Sureda, A. y Merlo, E. Z. (1980). Estudio sinóptico de los tisanópteros argentinos (Insecta). Obra del Centenario del Museo de La Plata, 6, 91-166.

Eichhorn, K. W. y Lorenz, D. H. (1977). Phenological DevelopmentStages of the Grapevine. Nachrichtenblatt des deutschen Pflanzenschutzdienstes, 29, 119-120.

Formolo, R., Rufato, L., Botton, M. y Machota, R. (2011). Diagnóstico da área cultivada com uva fina de mesa (Vitis vinifera L.) sob cobertura plástica e do manejo de pragas. Revista Brasileira de Fruticultura, 33(1), 103-110. https://doi.org/10.1590/S010029452011005000047

Hidalgo Fernández-Cano, L. e Hidalgo Togores, J. (2011). Tratado de Viticultura (4a ed.). Mundi Prensa.

Hoddle, M. S., Mound, L. A. y Paris, D. L. (2012). Thrips of California 2012. Queensland, Australia: CBIT Publishing.__ https://keys.lucidcentral.org/keys/v3/ thrips of california/authors/authors.html

Instituto Nacional de Viticultura (INV) (2020). Estadísticas vitivinicolas. Informes anuales Recuperado de https://www.argentina.gob.ar/inv/vinos/estadisticas/ superficie/anuarios

Jensen, F. y Luvisi, D. (1973). Flower thrips nymphs involved in scarring of Thompson seedless grapes. California Agriculture, 27(10), 8-9.

Kaplan, M., Bayhan, E. y Atakan, E. (2016). Determination of Thysanoptera species, their seasonal abundance and distribution in vineyard areas of Mardin Province. Turkish Bulletin of Entomology, 6(2), 161-168. http:// dx.doi.org/10.16969/teb.03520

Kirk, W. D. (1997). Feeding. En T. Lewis (Ed.), Thrips as Crop Pests (119-174). CAB International.

Kirk, W. D. (2 al 7 de julio de 2001). The pest and vector from the West:Frankliniella occidentalis. En R. Marullo y L. Mound (Eds.), Thrips and tospoviruses: Proceedings of the 7th International Symposium on Thysanoptera (pp. 33-42). Reggio Calabria, Italia. https://www.ento.csiro.au/thysanoptera/Symposium/ Section1/3-Kirk.pdf

Marroni, M., Teulon, D., Scott, R. y Jaspers, M. (2012). Abundance of Thrips obscuratus (Crawford) (Thysanoptera: Thripidae) during wine grape flowering in different regions in New Zealand. New Zealand Entomologist, 35(1), 10-13. https://doi.org/10.1080/00 779962.2012.649704

Merk, R., Schirra, K., Louis, F. y Zebitz, C. (2006). Thrips species and control of thrips (Thysanoptera: Thripidae) on grapevine in Rheinland-Pfalz. Mitteilungen der Deutscher Gessellschaftür Allgemeine und Angewardte Entomologie, 15, 277-280.

Moleas, T. y Addante, R. (1993). The most important thrips of curtain reared vineyards. Bio-ethological notes. Annali della Facolta di Agraria, Bari, 34, 203-207.

Monteiro, R. C. (1999). Estudos taxonômicos de tripes (Thysanoptera) constatados no Brasil, comêmfase no gêneroFrankliniella. (Tesis doctoral). Escuela Superior de Agricultura Luiz de Queiroz, Universidad de Sâo Pablo, Brasil. https://doi.org/10.11606/T.11.1999.tde20210104-193655

Monteiro, R. C. (2 al 7 de julio 2001). The Thysanoptera fauna of Brazil. En R. Marullo y L. Mound (Eds.), Thrips and Tospoviruses: Proceedings of the 7th International Symposium on Thysanoptera (325-340). Reggio Calabria, Italia. https://www.ento.csiro.au/ thysanoptera/Symposium/Section9/49-Monterio.pdf

Moreira, A. N., Vargas de Oliveira, J., de Morais Oliveira, J. E., de Souza G. M. M. y Oliveira Breda, M. (2014). Injuries caused by Frankliniella spp. (Thysanoptera: Thripidae) on seedless grapes. Ciência e Agrotecnologia, 38(4), 328-334. https://doi. org/10.1590/S1413-70542014000400002

Moreira, A. N., Vargas de Oliveira, J., de Morais Oliveira, J. E., Costa Oliveira, A. C. y de Souza, I. D. (2012). Variação sazonal de espécies de tripes em videira de acordo com sistemas de manejo e fases fenológicas. Pesquisa Agropecuária Brasileira, 47(3), 328-335. https://doi.org/10.1590/S0100-204X2012000300003

Mound, L. A. (2014). Austral Thysanoptera: 100 years of progress. Austral Entomology, 53, 18-25. https://doi. org/10.1111/aen.12054 
Mound, L. A. y Kibby, G. (1998). Thysanoptera: an identification guide. (2a ed.) CAB International.

Mound, L. A. y Marullo, R. (1996). The thrips of Central and South America: an Introduction (Insecta: Thysanoptera). En Memoirs on Entomology International (Vol. 6). Associated Publishers.

Mujica, M. V., Scatoni, I. B., Franco, J., Nuñez, S. y Bentancourt, C. M. (2007). Fluctuación poblacional de Frankliniella occidentalis (Pergande) (Thysanoptera: Thripidae) en Vitis vinífera L. CV. Italia en la zona sur de Uruguay. Boletín de Sanidad Vegetal Plagas, 33(4), 457-467.

Organización Internacional de la Viña y el Vino (OIV) (2018). Normas y documentos técnicos. https://www. oiv.int/es/normas-y-documentos-tecnicos/analisisestadisticos/informacion-estadistica

Ortiz, M. (1977). El género Frankliniella Karny (Thysanoptera: Thripidae) en el Perú. Revista Peruana de Entomología, 20(1), 49-62.

Özsemerci, F., Aksit, T. y Tunç, I. (2011). Composition, seasonal abundance and within-plant distribution of Thysanoptera species associated with seedless grapes (Sultana) in districts of Manisa, Turkey. Turkish Journal of Entomology, 35(1), 103-118.

Pavan, F., Bigot, G., Cargnus, E. y Zandigiacomo, P. (2014). Influence of the carpophagous generations of the European grapevine moth Lobesia botrana on grape bunch rots. Phytoparasitica 42, 61-69. https:// doi.org/10.1007/s12600-013-0338-5

Pearson, R. C. y Goheen, A. C. (2007). Plagas y enfermedades de la vid. Editorial Mundi-Prensa.

Pinent, S., Pinent, C., Botton, M. y Redaelli, L. (11 al 15 septiembre de 2005). Thrips species (Thysanoptera) on strawberry, persimmon and grape in the Gaúcho Highlands, Rio Grande do Sul State, southern Brazil. En D. Ullman, J. Moyer, R. Goldbach, G. Moritz (Eds.), VIII International Symposium on Thysanoptera and Tospoviruses (pp. 33). Pacific Grove, California,
EE. UU.: Journal of Insect Science. https://doi. org/10.1673/031.007.2801

Reitz, S. R. (2009). Biology and ecology of the western flower thrips (Thysanoptera: Thripidae): The Making of a Pest. Florida Entomologist, 92(1), 7-13. https://doi. org/10.1653/024.092.0102

Ripa, R., Rodriguez, F. y Vargas, R. (1992). Asociación entre trips (Thrips tabaci Lindeman y Frankliniella cestrum Moulton) durante la floración en uva de mesa y "russet" en la cosecha. I. Control químico. Agricultura Técnica, 52(3), 231-240.

Ripa, R., Rodriguez, F. y Vargas, R. (1993). Asociación entre thrips (Thrips tabaci Lindeman y Frankliniella cestrum Moulton) durante la floración en uva de mesa y russet en la cosecha. II Aspectosbiológicos. Agricultura técnica, 53(1), 16-22.

Soares de Matos, S. T., de Andrade, D. H., Paneghini Zanata, R. M., Azevedo, E. B. y Lima Bezerra, E. F. (2019). New records of thrips species on mango and natural enemies associated. Revista Brasileira de Fruticultura, 41(1), e-123. http://dx.doi. org/10.1590/0100-29452019123

Terry, L. I. (1997). Host selection, communication and reproductive behaviour. En T. Lewis (Ed.), Thrips as crop pests (65-118). CAB International.

Viglianco, A., de Borbón, C., Cragnolini, C., Bracamonte, E., Novo, R. y Tosutti, L. (6 al 8 de septiembre de 2005). Identificación de trips en cultivos de vid en Colonia Caroya, Córdoba. En XXVIII Congreso Argentino de Horticultura (pp.136). Río Negro, Argentina: Asociación Argentina de Horticultores.

Yokohama, V. Y. (1977 a). Frankliniella occidentalis and scars on table grapes. Environmental Entomology, 6(1), 25-30.

Yokoyama, V. Y. (1977 b). Drepanothrips reuteri on Thompson Seedless Grapes. Environmental Entomology, 6(1), 21-24. 